\title{
2019 Chinese Clinical Practice Guidelines for the Prevention of Mother-to-child Transmission of Hepatitis B Virus
}

\author{
Jinfeng Liu ${ }^{\# 1,2}$, Tianyan Chen ${ }^{\# 1,2}$, Yaolong Chen ${ }^{\# 3,4}$, Hong Ren*5, Guiqiang Wang*6, \\ Wenhong Zhang*7', Yingren Zhao*1,2' and Society of Infectious Diseases and Chinese Medical \\ Association
}

\begin{abstract}
${ }^{1}$ The First Affiliated Hospital of Xi'an Jiaotong University, Xi'an, Shaanxi, China; ${ }^{2}$ Shaanxi Clinical Research Center of Infectious Diseases, Xi'an, Shaanxi, China; ${ }^{3}$ Evidence-Based Medicine Center, Basic Medical Sciences, Lanzhou University, Lanzhou, Gansu, China; ${ }^{4}$ WHO Collaborating Centre for Guideline Implementation and Knowledge Translation, Lanzhou, Gansu, China;

${ }^{5}$ Department of Infectious Diseases, The Second Affiliated Hospital of Chongqing Medical University, Chongqing, China;

${ }^{6}$ Hepatology Center, Department of Infectious Diseases, Peking University First Hospital, Beijing, China; ${ }^{7}$ Department of
\end{abstract} Infectious Diseases, Huashan Hospital Affiliated to Fudan University, Shanghai, China

\begin{abstract}
To develop the evidence-based guidelines for managing mother-to-child transmission of hepatitis B virus in China, a multidisciplinary guideline development group was established. Clinical questions were identified from two rounds of surveys on the concerns of first-line clinicians. We conducted a comprehensive search and review of the literature. A grading of recommendations' assessment, development, and evaluation system was adopted to rate the quality of evidence and the strength of recommendations. Recommendations were formulated based on the evidence, overall balance of benefits and harms (at individual and population levels), patient/health worker values and preferences, resources available, cost-effectiveness, and feasibility. Eventually, recommendations related to 13 main clinical concerns were developed, covering diagnostic criteria, treatment indications, antiviral therapy choice, timing to initiate and discontinue treatment, immunoprophylaxis strategy at birth, and how to deal with special situations, such as unintended pregnancy, assisted reproduction, and breastfeeding. The guidelines are intended to serve as guidance for clinicians
\end{abstract}

Keywords: Hepatitis B virus; Mother-to-child transmission; Clinical practice guidelines; Prevention; GRADE.

Abbreviations: ADV, adefovir dipivoxil; AGREE II, Appraisal of Guidelines for Research and Evaluation; ALT, alanine aminotransferase; $\mathrm{CHB}$, chronic hepatitis $B$; $C K$, creatine kinase; ETV, entecavir; GRADE, grading of recommendations assessment, development, and evaluation; HBeAg, hepatitis B e antigen; HBIG, human hepatitis B immunoglobulin; HBsAg, hepatitis B surface antigen; HBV, hepatitis B virus; IFN, interferon; LAM, lamivudine; LdT, telbivudine; MTCT, mother-to-child transmission; PICOs, population, intervention, comparator, outcomes; RIGHT, Reporting Items for Practice Guidelines in Healthcare; TAF, tenofovir alafenamide; TDF, Tenofovir disoproxil fumarate; TFV, tenofovir; ULN, upper level of normal.

Received: 22 July 2020; Revised: 18 August 2020; Accepted: 8 September 2020 \#These authors contributed equally to this work.

* Correspondence to: Yingren Zhao, Department of Infectious Diseases, The First Affiliated Hospital of Xi'an Jiaotong University, Xi'an, Shaanxi 710061, China. Tel: +86-13509187086, Fax: +86-29-85324594, E-mail: zhaoyingren@mail.xjtu.edu.cn; Hong Ren, Department of Infectious Diseases, The Second Affiliated Hospital of Chongqing Medical University, Chongqing 400010, China. E-mail: renhong0531@vip.sina.com.cn; Guiqiang Wang, Hepatology Center, Department of Infectious Diseases, Peking University First Hospital, Beijing 100034, China. E-mail: john131212@sina.com; Wenhong Zhang, Department of Infectious Diseases, Huashan Hospital Affiliated to Fudan University, Shanghai 200040, China. E-mail: zhangwenhong@fudan.edu.cn and patients, to optimize the management of majority of pregnant women who are positive for hepatitis B surface antigen. Guideline registration: International Practice Guide Registration Platform (IPGRP-2018CN040).

Citation of this article: Liu J, Chen T, Chen Y, Ren $\mathrm{H}$, Wang G, Zhang W, et al. 2019 Chinese clinical practice guidelines for the prevention of mother-to-child transmission of hepatitis $B$ virus. J Clin Transl Hepatol 2020;8(4):397-406. doi: 10.14218/JCTH.2020.00070.

\section{Introduction}

As a primary cause of liver cirrhosis and cancer, chronic hepatitis $B(\mathrm{CHB})$, accounting for about 1 million deaths per year, remains a severe public health problem and presents a heavy disease burden and economic burden to society and families. ${ }^{1}$ With an extensive hepatitis $B$ vaccination program implemented, mother-to-child transmission (MTCT) has become the key obstacle to realizing the World Health Organization's goal of reducing the prevalence of hepatitis B surface antigen (HBsAg) among children aged 5 , to $0.1 \%{ }^{2}$ Furthermore, MTCT is responsible for familial clustering of hepatitis $B$ virus (HBV) infection ${ }^{3}$ in which the risk of cirrhosis and hepatocellular carcinoma increase significantly and the age of onset of end-stage liver diseases was advanced dramatically. ${ }^{4}$ Elimination of MTCT is crucial to decreasing new HBV infections and to minimizing the burden of HBV-related diseases.

As the most principal strategy to prevent new HBV infections, the hepatitis vaccine has reduced the rate of MTCT by more than $80 \%{ }^{5}$ Whereas, among infants born to hepatitis $\mathrm{B}$ e antigen ( $\mathrm{HBeAg}$ )-positive mothers, there are still $8 \%$ becoming $\mathrm{CHB}$ after vaccine immunoprophylaxis, and $4 \%$ after immmunoprophylaxis of vaccine combined with human hepatitis B immunoglobulin (HBIG). ${ }^{5}$ Annually, there are almost 2 million new infections in children younger than 5 years. Antiviral intervention during pregnancy has been widely adopted to interrupt MTCT; nevertheless, there is still controversy about treatment indications, antiviral therapy choice, and the timing to initiate and discontinue the treatment, and so on. 
To further standardize the clinical recommendations for top concerns of first-line clinicians, a multidisciplinary guideline development group was established to comprehensively evaluate the evidence and overall balance of benefits and harms, while the guidelines do not cover the whole spectrum of prevention and treatment of MTCT. As with clinical practice guidelines, they provide general guidance to optimize management of the majority of pregnant patients infected with HBV, while clinical judgement considering a unique patient and reliability of clinical care should be considered. In addition, despite accumulated knowledge, areas of uncertainty still exist and therefore health care workers, patients, and public health authorities must continue to make choices based on evolving evidence. The guidelines have two versions: the Chinese language version published in the Chinese Journal of Infectious Diseases and the English language version, which is the current version.

\section{Methods}

The guidelines were launched by the Society of Infectious Diseases, Chinese Medical Association, supported by the Chinese Grading of Recommendations Assessment, Development and Evaluation (GRADE) Center in methodology, and developed according to the World Health Organization's Handbook for Guideline Development (2014). ${ }^{6}$ Appraisal of Guidelines for Research and Evaluation (known as AGREE II $^{7}$ and Reporting Items for Practice Guidelines in Healthcare (known as RIGHT) tool ${ }^{8}$ were also referred to. Three groups were established for developing the guidelines: steering committee, guidelines development panel, and guidelines secretary group. The steering committee consisted of 3 well-known experts in the field, with the following missions: 1) approve the use of population, intervention, comparator, outcomes (PICOs), 2) supervise the literature search and systematic reviews, 3) check the grade of the evidence, 4) finalize the recommendations using a modified Delphi approach, and 5) approve the publication of the guidelines. A multidisciplinary guidelines development panel, including experts from across the country in infectious diseases, hepatology, obstetrics, pediatrics, and methodology, was established, and tasked with the following missions: 1) generate the scope of the guideline and draft the PICOs, 2) grade the quality of the evidence, 3) draft the preliminary recommendations, and 4) write and publish the draft guideline. The guidelines secretary group conducted systematic reviews and investigated patients' views and preferences. All members involved in guidelines development were required to disclose any potential conflicts of interest, which were reviewed by the chairs (Yingren Zhao and Yaolong Chen). No relevant conflict of interest was reported.

Before initiating the guidelines, we wrote the protocol and registered it in the International Practice Guidelines Registry Platform (http://www.guidelines-registry.org, IPGRP2018CN040). First, we collected questions reflecting clinicians' concerns through two rounds of questionnaire survey. Two hundred sixty-one copies of the questionnaire were collected from 98 facilities across mainland China, covering 26 provinces, municipalities, and autonomous regions. After deduplication and combination, 16 PICO questions were identified from among 68 clinical questions, based on importance grade. Published articles and conference abstracts were identified from PubMed, Embase, the Cochrane Library, and three Chinese literature databases (CNKI, WanFang, and CBM). The evidence synthesis group conducted systematic reviews and other literature searches for each question. We finally conducted 11 new systematic reviews. The GRADE was used to evaluate and rate the quality of evidence body (Table 1$).{ }^{9}$ We then formulated recommendations and rated their strengths after comprehensive assessment of the quality of evidence, consideration of the overall balance of benefits and harms, patient/health worker values and preferences, cost-effectiveness, and feasibility. Finally, the guidelines development panel reached a consensus on each recommendation based on three rounds of Delphi survey and also reached a consensus on management algorithm for MTCT of HBV (Fig. 1). A flow chart describes the process of the guidelines development (Fig. 2).

\section{Recommendations}

The guidelines contain 24 recommendations on the top 13 concerns of clinicians, covering diagnostic criteria, monitoring and prevention during pregnancy, and breastfeeding, as well

Table 1. Grades of evidence and recommendations

\begin{tabular}{|c|c|}
\hline Grade of evidence & Notes \\
\hline High quality (A) & $\begin{array}{l}\text { Further research is very unlikely to } \\
\text { change our confidence in the } \\
\text { estimate of effect. }\end{array}$ \\
\hline $\begin{array}{l}\text { Moderate quality } \\
\text { (B) }\end{array}$ & $\begin{array}{l}\text { Further research is likely to have an } \\
\text { important impact on our confidence } \\
\text { in the estimate of effect and there is } \\
\text { a possibility that it may change the } \\
\text { estimate. }\end{array}$ \\
\hline Low quality (C) & $\begin{array}{l}\text { Further research is very likely to } \\
\text { have an important impact on our } \\
\text { confidence in the estimate of effect } \\
\text { and may be substantially different } \\
\text { from the estimate of the effect. }\end{array}$ \\
\hline $\begin{array}{l}\text { Very low quality } \\
\text { (D) }\end{array}$ & $\begin{array}{l}\text { The estimate of effect is very } \\
\text { uncertain, and the true effect is } \\
\text { likely to be substantially different } \\
\text { from the estimate of effect. }\end{array}$ \\
\hline \multicolumn{2}{|l|}{$\begin{array}{l}\text { Grade of } \\
\text { recommendation }\end{array}$} \\
\hline Strong (1) & $\begin{array}{l}\text { The Guideline Panel is confident that } \\
\text { the desirable effects of an } \\
\text { intervention outweigh its } \\
\text { undesirable effects (strong } \\
\text { recommendation for an } \\
\text { intervention), or that the } \\
\text { undesirable effects of an } \\
\text { intervention outweigh its desirable } \\
\text { effects (strong recommendation } \\
\text { against an intervention). }\end{array}$ \\
\hline Weak (2) & $\begin{array}{l}\text { The desirable effects probably } \\
\text { outweigh the undesirable effects } \\
\text { (weak recommendation for an } \\
\text { intervention) or undesirable effects } \\
\text { probably outweigh the desirable } \\
\text { effects (weak recommendation } \\
\text { against an intervention) but less } \\
\text { uncertain higher cost or resource } \\
\text { consumption exists. }\end{array}$ \\
\hline
\end{tabular}




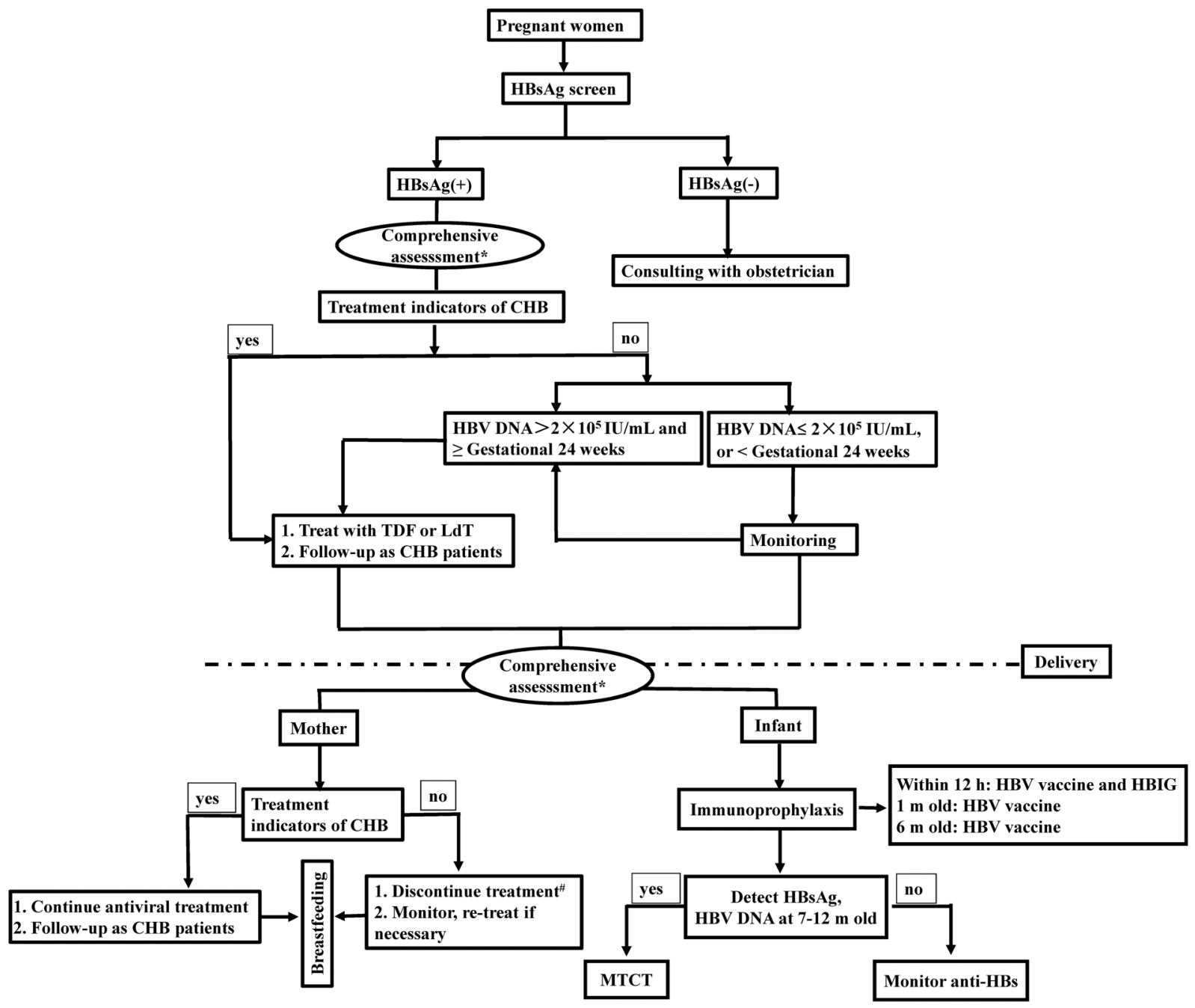

Fig. 1. Management algorithm for mother-to-child transmission of hepatitis B virus. *Comprehensive assessment: liver biochemical function, HBV DNA, imaging assessment; "Time to discontinue treatment: at delivery, postpartum 1 or 3 m old.

Abbreviations: CHB, chronic hepatitis B infection; HBIG, human hepatitis B immunoglobulin; HBsAg, hepatitis B surface antigen; HBV, hepatitis B Virus; m, months; MTCT, mother-to-child transmission; TDF, tenofovir disoproxil fumarate; LdT, telbivudine.

as immunoprophylaxis strategy at birth. All the recommendations are as follows.

\section{Question 1: How to diagnose MTCT of HBV?}

Recommendation: Infants with HBsAg and/or HBV DNA positive at 7-12 months-old are diagnosed as having CHB infection due to MTCT (1B).

\section{Recommendation evidence}

The previous reported rate of MTCT varied markedly, owing to inconsistence in specimens, timepoints of detection, and diagnostic criteria. Currently, HBsAg and/or HBV DNA positive at 712 months-old is deemed as having obtained CHB transmitted from mothers. ${ }^{10,11}$ Whereas, there is no systematic review to assess the criterion. A systematic review and network metaanalysis showed the rate of HBsAg and/or HBV DNA positive at birth in cord blood or venous blood was significantly higher than that at 6,7 , or 12 months-old, ${ }^{12}$ which indicated the excessive positive rate at birth may be attributed to false positivity caused by contamination of maternal blood or transient viremia due to placental abruption at birth. ${ }^{13,14}$ In addition, there was no significant difference among the positive rates at age of 6,7 and 12 months. ${ }^{12}$ Therefore, HBV serological markers and HBV DNA should be tested at 7-12 months-old, namely 1-6 months after three dosages of vaccination, to determine the immune results and infection status. Moreover, the infants over 12 months-old with HBsAg and/or HBV DNA positive at first visit are also supposed to acquire HBV infection by MTCT.

Question 2: What is the vaccination schedule for infants of $\mathrm{HBsAg}(+)$ mothers?

Recommendations:

2.1: The infants of $\mathrm{HBsAg}(+)$ mothers should receive hepatitis B vaccine and 100 IU HBIG within $12 \mathrm{~h}$ after birth, and the following two doses of vaccine at 1 and 6 months-old, respectively (1A).

2.2 For premature or low-birth weight infants, the combined immunoprophylaxis should be administered within $12 \mathrm{~h}$ after birth when the vital signs are stable or after the 
Liu J. et al: Guidelines for prevention of MTCT of HBV

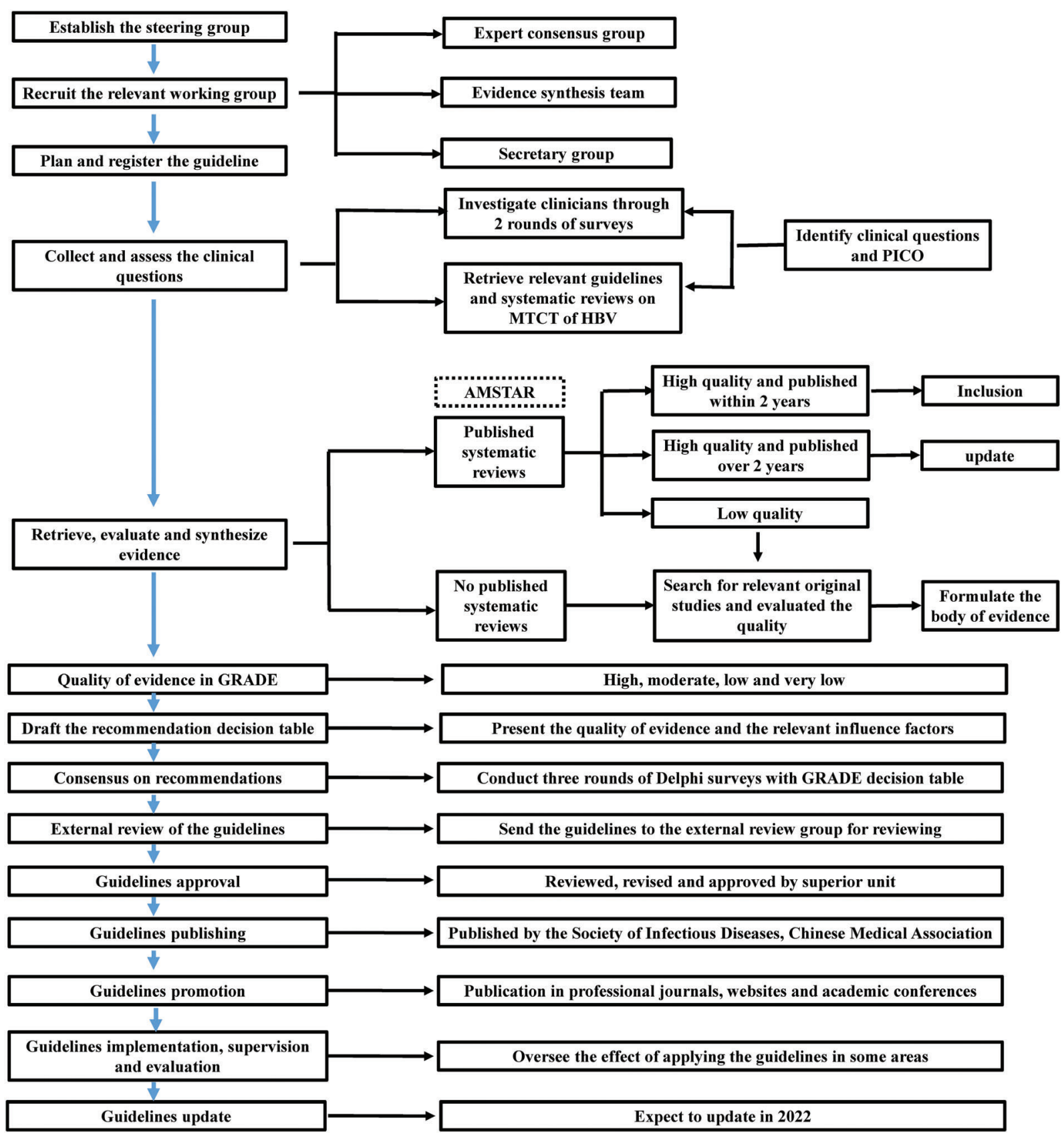

Fig. 2. Flowchart of the process of the guidelines development.

Abbreviations: GRADE, the Grading of Recommendations Assessment, Development and Evaluation; HBV, hepatitis B virus; MTCT, mother-to-child transmission; PICO, population, intervention, comparator, outcomes.

\section{viral signs become stable. Three doses of full-course vaccine should be administered subsequently (1A).}

\section{Recommendation evidence}

Combined immunoprophylaxis is the current standard immunoprophylaxis strategy for preventing MTCT in $\mathrm{HBsAg}(+)$ mothers. Several sets of guidelines ${ }^{15-19}$ recommend newborns of $\mathrm{HBsAg}(+)$ mothers receive hepatitis $B$ vaccine
(10 $\mu \mathrm{g}$ recombinant yeast-derived hepatitis $B$ vaccine or 20 $\mu \mathrm{g}$ recombinant Chinese hamster ovary cells hepatitis $B$ vaccine) and HBIG, and vaccines are administered at 0,1 , and 6 months-old, respectively. For the premature or lowbirth weight infants, one dose of vaccine is implemented as soon as possible within $12 \mathrm{~h}$ after birth (when the viral signs are stable) and another three doses of full-course vaccine are conducted after 1 month-old age is reached. For the very lowbirth weight infants, those with severe birth defects, severe 
asphyxia, or respiratory distress syndrome, should receive four doses of vaccine, administered after the vital signs become stable. By systematic review, we found 200 IU HBIG shows equivalent preventive effectiveness with $100 \mathrm{IU}$ HBIG in infants born to CHB mothers (relative risk: 1.08 , (0.64-1.82)) and $\mathrm{HBeAg}(+)$ mothers (relative risk: 0.84 $(0.39-1.77)) .{ }^{20}$ Considering cost-effectiveness, 100 IU HBIG is recommended to newborns of $\mathrm{HBsAg}(+)$ mothers.

Whether vaccine should be boosted in infants born to $\mathrm{HBsAg}(+)$ mothers is unclear. Systematic review also showed that the response to vaccine is similar between infants born to $\mathrm{HBsAg}(+)$ mothers and the general population. ${ }^{21}$ While the titer of anti-hepatitis $B$ surface antibody should be regularly assessed, boost could be considered when the titer is less than $10 \mathrm{IU} / \mathrm{mL}$, regarding the high-risk circumstances of infection.

Question 3: What is the threshold of HBV DNA for antiviral intervention during pregnancy?

Recommendations:

3.1 Antivirals should be recommended to pregnant women with HBV DNA $>2 \times 10^{5} \mathrm{IU} / \mathrm{mL}$ (1B).

3.2 Antiviral intervention could be decided after discussion with pregnant women with HBV DNA of $1 \times 10^{4}-2 \times 10^{5} \mathrm{IU} / \mathrm{mL}$ (2C).

\section{Recommendation evidence}

Maternal high viremia is an independent risk factor for MTCT. ${ }^{22}$ The majority of guidelines from associations for the study of liver diseases, such as the American Association for the Study of Liver Diseases, the European Association for the Study of the Liver, the Asian Pacific Association for the Study of the Liver, and the National Institute for Health and Care Excellence, recommend antiviral intervention during pregnancy for preventing MTCT, whereas, the threshold of HBV DNA ranges from $2 \times 10^{5} \mathrm{IU} / \mathrm{mL}$ to $10^{7} \mathrm{IU} / \mathrm{mL} .{ }^{17,23-25} \mathrm{In}$ addition, the recommendations are mainly based on clinical trials aiming to evaluating efficacy of antivirals ${ }^{11,26}$ or single retrospective cohort investigation. 27,28

A systematic review including 6027 patients from 18 studies indicated the rates of MTCT were $0,0.88 \%, 1.15 \%$, $4.81 \%, 10.04 \%$ and $18.80 \%$ in pregnant women with antenatal HBV DNA $<1 \times 10^{4} \mathrm{IU} / \mathrm{mL}, 1 \times 10^{4} \mathrm{IU} / \mathrm{mL}-1 \times 10^{5} \mathrm{IU} / \mathrm{mL}$, $1 \times 10^{5} \mathrm{IU} / \mathrm{mL}-1 \times 10^{6} \mathrm{IU} / \mathrm{mL}, 1 \times 10^{6} \mathrm{IU} / \mathrm{mL}-1 \times 10^{7} \mathrm{IU} / \mathrm{mL}$, $1 \times 10^{7} \mathrm{IU} / \mathrm{mL}-1 \times 10^{8} \mathrm{IU} / \mathrm{mL}$, and $>1 \times 10^{8} \mathrm{IU} / \mathrm{mL}$, respectively. ${ }^{5}$ The pooled rate of MTCT was as high as $10 \%$ in women with antenatal HBV DNA $\geq 1 \times 10^{6} \mathrm{IU} / \mathrm{mL}$, which was remarkably higher than those with HBV DNA $<1 \times 10^{6} \mathrm{IU} / \mathrm{mL}$. Antivirals should be recommended for these pregnant women. Pregnant women with HBV DNA of $1 \times 10^{4} \mathrm{IU} / \mathrm{mL}-$ $2 \times 10^{5} \mathrm{IU} / \mathrm{mL}$ still carry risk for transmitting the virus to their infants. Considering the high prevalence of HBsAg in China, antiviral intervention during pregnancy could be suggested in those with family history of HBV infection or history of MTCT. The benefit of antiviral treatment in terms of protecting newborns from HBV infection and controlling hepatitis activity in pregnant women with hepatitis should be explained before administration. At the same time, the patients should be notified of the side effects of antivirals, drug-resistant mutations, potential harms to the fetus, and hepatic flare after antivirals discontinuation, and so on.
Question 4: Which antivirals should be recommended for preventing MTCT?

Recommendations:

4.1. Tenofovir disoproxil fumarate (TDF) or telbivudine (LdT) is recommended for pregnant women with HBV DNA $>2 \times 10^{5} \mathrm{IU} / \mathrm{mL}$ (1B).

4.2 TDF is preferred in pregnant women with drugresistance to lamivudine (LAM) or LdT (2C).

\section{Recommendation evidence}

TDF and LdT do not show reproductive toxicity in animal experiments. ${ }^{29,30}$ Plenty studies have manifested undifferentiated effectiveness of TDF and LdT in preventing MTCT. ${ }^{10,11,31}$ In addition, the frequency of adverse events in pregnant women receiving TDF or LdT, such as abnormal creatinine, postpartum hemorrhage, rate of cesarean section, birth defects, and preterm birth were comparable with general population. ${ }^{26} \mathrm{~A}$ prospective cohort has demonstrated undifferentiated growth and development in infants born to LdT-treated pregnant women during 5 years' follow-up. ${ }^{32}$ For the pregnant women at high risk of MTCT, TDF or LdT should be administered to inhibit virus replication and reduce the transmission risk.

LAM and LdT present high potential of drug-resistance. Previous studies have confirmed the advantage of TDF over LAM and LdT as antiviral therapy. ${ }^{33}$ Furthermore, the superiority of TDF has also been demonstrated in pregnant women with LAM or LdT-resistant mutants. ${ }^{34}$ Hence, TDF could be suggested to pregnant women with drug-resistance to LAM or LdT.

As the first-line antiviral medicine for CHB patients, tenofovir alafenamide (TAF) has no influence on reproductive function in animal experiments. A study in human immunodeficiency virus-infected pregnant women assessed the safety of TAF but had small sample size. ${ }^{35}$ The undergoing prospective, multicenter clinical trials will provide evidence for efficacy and safety of TAF in pregnant women with CHB.

Question 5: When should the antiviral be initiated to prevent MTCT during pregnancy?

Recommendation: The antiviral should be initiated at 24-28 weeks of gestation for preventing MTCT (2C).

\section{Recommendation evidence}

Head-to-head comparison of the efficacy and safety of different timepoints to initiate antiviral intervention is lacking. A Bayesian network meta-analysis and system review showed that the risk of MTCT decreased significantly in pregnant women accepting intervention before 28 weeks of gestation, as compared to those initiating after 28 weeks (relative risk: 0.019 , (0.00034-0.19)). ${ }^{36}$ Additionally, the efficacy and safety of antiviral therapy initiated from 24 weeks of gestation have been identified in cohort studies and case-control studies. ${ }^{10,11,31,37,38}$ Therefore, pregnant women with high HBV DNA levels $\left(>2 \times 10^{5} \mathrm{IU} / \mathrm{mL}\right.$ ) are recommended to initiate antiviral intervention at 24-28 weeks of gestation. For pregnant women with high viremia who are visiting the hospital after 28 weeks of gestation, antiviral intervention should be initiated immediately. For pregnant women with HBV DNA of $1 \times 10^{4} \mathrm{IU} / \mathrm{mL}-2 \times 10^{5} \mathrm{IU} / \mathrm{mL}$ who agree to take antivirals, the intervention could be initiated no later than 28 weeks of gestation. 
Question 6: How to manage unintended pregnancy during antiviral therapy?

Recommendation: For patients who become unintentionally pregnant during antiviral therapy, TDF or LdT treatment should be continued (2B); adefovir dipivoxil (ADV) or entecavir (ETV) should be switched to TDF (2C); the potential risks of interferon (IFN) should be fully informed, and the patient should switch to TDF if the patients and/or family members decide to carry on the pregnancy $(2 \mathrm{C})$.

\section{Recommendation evidence}

Data from the Antiretroviral Pregnancy Registry and wellcontrolled studies have revealed superior safety of TDF and LdT in pregnant women. ${ }^{10,11,31}$ Additionally, TDF shows great advantage in antiviral treatment because of superior resistance profile and more extensive safety data in pregnant women. A systematic review ${ }^{39}$ showed the rate of birth defects as $0.66 \%$ in pregnant women exposed to nucleos $(t)$ ide analogues, which are undifferentiated from the rate in Chinese population $(5.6 \%)^{40}$ and Metropolitan Atlanta Congenital Defects Program (2.8\%). ${ }^{41}$ Therefore, childbearing women with unintended pregnancy during antiviral therapy should continue TDF or LdT.

The safety of ADV and ETV in pregnancy has not be elucidated clearly. A systematic review ${ }^{39}$ including safety data from the Antiretroviral Pregnancy Registry showed the rate of birth defects in pregnant women exposed to ADV or ETV is comparable with that among the general population. Hence, women undergoing treatment of ADV or ETV could continue a pregnancy under doctors' guidance. However, regarding the risks of birth defects associated with high dose of ADV or ETV in animal experiments, switching to TDF is recommended.

We found low-quality evidence about the safety of IFN during pregnancy. Randomized controlled study of IFN administration during pregnancy is unlikely to be conducted, given the ethical concerns of such a trail. Guidelines suggest that IFN is contraindicated during pregnancy and contraception is recommended during IFN treatment, ${ }^{24,42,43}$ while how to deal with unintended pregnancy during IFN treatment causes substantial controversy between obstetricians and hepatologists. In a Rhesus monkey model, 90-180 times the recommended dosage of IFN led to increased rate of abortion. A series of cohort studies had displayed undifferentiated rates of adverse effects, including birth defects in pregnant women with essential thrombocythemia ${ }^{44,45}$ or multiple sclerosis ${ }^{46,47}$ following exposure to INF. In addition, in case reports, the infants born to HBV or hepatitis C virus/human immunodeficiency virus-infected mothers exposed to IFN during first trimester did not display abnormal rates of birth defects. ${ }^{4-50}$ The data from Bayer HealthCare's global pharmacovigilance database have not revealed an obvious increase of adverse effects in pregnant women exposed to IFN during the early trimester. ${ }^{47,51}$ With comprehensive assessment of toxicology, clinical reports, and views of obstetric experts, we suggest the risk of IFN should be fully informed to the pregnant women and their family members, and the TDF should be recommended instead of IFN if the family decides to continue the pregnancy.

Question 7: How to deal with HBsAg(+) pregnant women with hepatic flare?
Recommendations:

7.1: For pregnant women with HBV DNA $\leq 2 \times 10^{5} \mathrm{IU} /$ $\mathrm{mL}$ and $A L T<2 \times$ the upper limit of normal (ULN), close monitoring should be conducted (2C).

7.2: If a hepatic flare is confirmed to be associated with immune activation, antiviral treatment and monitoring should be initiated as CHB patients (1C).

\section{Recommendation evidence}

We found low-quality evidence on management of hepatic flare during pregnancy. About $10 \%$ of pregnant women presents hepatic flare and the majority of those cases involve mild ALT elevation. 52,53 For pregnant women with HBV DNA $\leq 2 \times 10^{5} \mathrm{IU} / \mathrm{mL}$, mild ALT elevation ( $\left.<2 \times U L N\right)$ and no cirrhosis, we suggest close monitoring without antiviral treatment, according to existing evidence. ${ }^{52-54}$ A proportion of CHB patients with mild ALT flare experience disease progression. ${ }^{24}$ More evidence is required for treatment consideration in pregnant women with mild hepatitis flare.

For pregnant women with $2 \times U L N \leq A L T<5 \times U L N$, close monitoring is recommended. If ALT continues fluctuating and hepatitis is attributed to immune activation, a treatment decision should be made. ${ }^{24,55}$ Pregnant women with advanced fibrosis or cirrhosis should initiate antiviral treatment immediately, and close monitoring is required throughout the pregnancy.

Question 8: Which indicators should be monitored during antiviral therapy for pregnancies?

Recommendations:

8.1: For pregnant women taking antivirals to prevent MTCT, tests of liver biochemical function and HBV DNA should be conducted after 4 weeks (2C).

8.2: For those with hepatitis flares, more frequent monitoring and follow-up is recommended (2D). Renal function and serum phosphorus should be examined in patients receiving TDF, and creatine kinase (CK) should be measured in patients receiving LdT (2C).

\section{Recommendation evidence}

There is no consensus on monitoring timepoint and indicators examined in pregnant women undergoing antiviral treatment. A systematic review ${ }^{56}$ showed a mean decrease of $3.16 \log _{10}$ $\mathrm{IU} / \mathrm{mL}$ (95\% confidence interval: $2.97-3.35$ ) in HBV DNA after 4 weeks of antiviral treatment; therefore, HBV DNA level after 4 weeks of treatment can be tested to forecast the risk of transmission. HBV DNA levels should be tested again before delivery to further assess the risk.

There is potential influence on renal function and bone turnover $^{57}$ during long-term TDF treatment and risk of CK increase during LdT treatment. ${ }^{58}$ In terms of the potential adverse effects, renal function and serum phosphorus should be examined in pregnant women with TDF treatment and CK during LdT treatment.

Question 9: Does HBV infection influence assisted reproduction?

Recommendation: Considering the comparable cleavage rate, embryo implantation rate, pregnancy rate, and abortion rate in infertile women with $\mathrm{CHB}$, assisted reproduction could be conducted following the same 
intervention and monitoring algorithm as in other CHB pregnant women (2C).

\section{Recommendation evidence}

The impact of HBV on assisted reproduction and pregnancy outcomes is uncertain. Studies about MTCT rate in infertile women are scarce. One case-control study ${ }^{59}$ showed lower rate of fertilization, cleavage, high-quality embryos, and pregnancy in infertile women with HBV infection. In women with HBV DNA $\geq 5 \times 10^{2} \mathrm{IU} / \mathrm{mL}$, few investigations have shown that ovarian reserve was lower and the rate of fertilization and high-quality embryos was decreased. ${ }^{60,61}$ Nevertheless, the systematic review we performed showed the rate of fertilization to be only a little lower, while there were not significant differences in the rate of cleavage, high-quality embryos, implantation, pregnancy, and abortion. ${ }^{62}$ In these circumstances, the infertile women with HBV infection could accept assisted reproduction. The intervention strategy should follow the recommendations for $\mathrm{CHB}$ pregnant women.

Question 10: Could amniocentesis be conducted in CHB pregnant women?

Recommendations:

10.1: Amniocentesis can increase the risk of MTCT in pregnant women with $\mathrm{HBV}$ DNA $\geq 1 \times 10^{6} \mathrm{IU} / \mathrm{mL}$ and can be conducted only if the potential benefit is considered definite after assessment by an obstetrician (2B). 10.2: Amniocentesis is feasible after weighing the benefits and harms in pregnant women with HBV DNA $<1 \times 10^{6} \mathrm{IU} / \mathrm{mL}$ (2B).

\section{Recommendation evidence}

A previous systematic review ${ }^{63}$ concluded that the risk of HBV transmission in amniocentesis was low in women with HBV DNA $<1 \times 10^{6} \mathrm{IU} / \mathrm{mL}$, whereas the risk increased significantly in $\mathrm{HBeAg}(+)$ mothers with $\mathrm{HBV}$ DNA $\geq 1 \times 10^{6} \mathrm{IU} / \mathrm{mL}$ (relative risk: 3.41-9.54). The 2018 updated American Association for the Study of Liver Diseases Guidelines recommend the risk of MTCT be considered when assessing the potential benefit of amniocentesis in women with high viremia. ${ }^{17}$ Therefore, for pregnant women with low viral load, amniocentesis could be conducted with signed written content; while for the women with high viremia $\left(\geq 1 \times 10^{6} \mathrm{IU} / \mathrm{mL}\right)$, the risk of MTCT should be assessed thoroughly and amniocentesis could be conducted for screening inherited and chromosomal diseases after consultation with an obstetrician.

\section{Question 11: What is the influence of delivery mode on} MTCT?

Recommendation: Cesarean section may reduce the risk of MTCT in pregnant women with antenatal HBV DNA $>2 \times 10^{5} \mathrm{IU} / \mathrm{mL}$ without intervention during pregnancy, and could be considered when there is fetal distress, macrosomia, or overdue pregnancy (2C).

\section{Recommendation evidence}

With appropriate intervention and close monitoring, the risk of MTCT has decreased by a great degree, and the delivery mode does not affect MTCT. However, a portion of pregnant women do not undertake regular follow-up and appreciate intervention during pregnancy, especially in underdeveloped regions, in this case, cesarean section could reduce the risk of MTCT (relative risk: $0.41,95 \%$ confidence interval: $0.25 \sim 0.67, p<0.001)$ in the pregnant women with antental HBV DNA $>2 \times 10^{5} \mathrm{IU} / \mathrm{mL} .^{63}$ This population could benefit from cesarean section for reducing MTCT. After comprehensive assessment of the evidence and standpoints expressed by obstetricians, we suggest that cesarean section may be considered when there is fetal distress, macrosomia, or overdue pregnancy. To prevent excessive cesarean section, obstetric indications should also be followed.

Question 12: When should the antiviral be discontinued after delivery?

Recommendations:

12.1: Pregnant women taking antivirals for preventing MTCT can discontinue antiviral treatment immediately after delivery, 4 weeks postpartum, or 12 weeks postpartum (2C), and should be monitored closely for hepatitis flare and rebound of HBV DNA (2C).

12.2: Pregnant women accepting antivirals owing to hepatic flare should be monitored and treated according to guidelines for CHB patients after delivery (2D).

\section{Recommendation evidence}

There are a series of changes to the immune system and body function during pregnancy. No consensus has been reached about the timepoint to discontinue antiviral treatment because of the insufficient evidence on this issue. ${ }^{17,24,64}$ Previous studies determined that about $20 \%$ of parturient women present ALT flare, regardless of antiviral treatment or not, and that there are two flare peaks, at 1 month postpartum and 3 months postpartum; the majority recover spontaneously. ${ }^{52,53,65,66}$ The net-meta analysis showed no difference in rate of hepatitis flare among mothers discontinuing antiviral treatment immediately after delivery, 4 weeks postpartum, and 12 weeks postpartum, and those without antiviral intervention during pregnancy. ${ }^{67}$ In view of this, the pregnant women receiving antivirals for interrupting MTCT can discontinue treatment immediately after delivery, 4 weeks postpartum, or 12 weeks postpartum. HBV DNA could rebound after antiviral discontinuation; close monitoring should be conducted and re-antiviral treatment can be considered when meeting treatment indications for CHB therapy. ${ }^{24,55}$

Previous studies of women who manifested ALT flare during pregnancy identified the occurrence as a risk factor for postpartum hepatic flare ${ }^{66}$ and severe hepatitis has also been reported. ${ }^{68}$ Therefore, it is suggested that pregnant women with active hepatitis should undergo monitoring and continue treatment after delivery, following guidelines for $\mathrm{CHB}$ patients.

Question 13: Could CHB mothers breastfeed? Recommendations:

13.1: Breastfeeding is recommended after newborns accepting HBV vaccine and HBIG (2B).

13.2: The mother undergoing TDF treatment could give breastfeeding (2C).

\section{Recommendation evidence}

The research about breastfeeding in $\operatorname{HBsAg}(+)$ mothers is insufficient. One case-control study reported that the viral load in breast milk was related with maternal viral load ${ }^{69}$ while the risk of transmission did not increase with high 
maternal viremia. ${ }^{70,71}$ Further systematic review found that the risk of MTCT did not increase in infants accepting breastfeeding (relative risk: $0.73, p=0.21$ ).$^{72}$ Considering the significant benefit identified, breastfeeding is recommended to infants who undergo combined immunoprophylaxis.

The concentration of tenofovir (TFV) in breast milk and infants were $0.03 \%$ and $0.01 \%$ of recommended dose for infants from mothers with human immunodeficiency virus, respectively. ${ }^{73-75}$ In an investigation with small size of $\mathrm{HBsAg}$ (+) mothers undergoing TDF treatment, the TFV was undetectable in infants accepting breastfeeding. In addition, TFV cannot be absorbed via gastrointestinal tract. Hence, the mothers undergoing TDF treatment after delivery could give breastfeeding. Studies on infants accepting breastfeeding from LdT-treated mothers are scarce.

\section{Conclusions}

Clinical guidelines are derived from clinical concerns and are intended to direct practice. Major guidelines of prevention and treatment of $\mathrm{CHB}$ provide recommendations for pregnant women as a special population. ${ }^{17,23,24,55}$ The purpose of these guidelines is to provide scientific and specific guidance for the management of MTCT of HBV. Based on current clinical research outcomes, a preliminary exploration of the standardizations for managing MTCT has been established. Substantial relevant research evidence from China and other countries was fully retrieved and evaluated. Focusing on pregnant women with $\mathrm{CHB}$, an expert panel from multiple disciplines proposed recommendations for the top 13 clinical concerns. Following accumulation of additional evidence and research findings, we plan to update the guidelines (at minimum) within 5 years of this publication (estimated 2022).

There are inevitably limitations to the process of guidelines' development that mainly reflect the low quality of the existing clinical studies and the small number of rigorously designed and implemented randomized controlled trials in this special population. For ethical issues, there is a lack of clinical trials on monitoring and treating $\mathrm{CHB}$ pregnant women with hepatitis flare; therefore, weak recommendations were proposed. In addition, scarce evidence exists on the rate of MTCT in infertile women with assisted reproduction, limiting the strength of recommendations. For infants born to CHB mothers, it is still essential to evaluate the necessity of booster vaccination. However, further scientific research will gradually address these shortcomings. Beyond that, as principal strategy, coverage of screening for HBsAg $(+)$ in childbearing women and the vaccination program requires more efforts.

Development of these guidelines is a small step toward the goal of standardized diagnosis and optimized management of MTCT. It is hoped that these guidelines will facilitate clinical research, accumulate more high-quality evidence in the future, and ultimately promote the elimination of MTCT.

\section{Members of the Guidelines Steering Committee Taisheng Li, Guiqiang Wang, Kehu Yang}

\section{Members of the Guidelines Development Panel}

Xuefan Bai, Hong Chen, Suhua Chen, Tianyan Chen, Xinyue Chen, Yaolong Chen, Mingliang Cheng, Xiaoguang Du, Xiangchun Ding, Shangrong Fan, Xuegong Fan, Zhiliang Gao, Yingli He, Peng Hu, Yanhong Huang, Yan Huang, Fanpu
Ji, Zhansheng Jia, Jianning Jiang, Peiru Jiang, Chengzhong Li, Chunfang $\mathrm{Li}$, Fen $\mathrm{Li}$, Jun $\mathrm{Li}$, Ke Li, Xuelan Li, Yongguo $\mathrm{Li}$, Zhengwen Liu, Qing Mao, Qinghua Meng, Qin Ning, Hong Ren, Hong Tang, Feng Wang, Jiuping Wang, Kai Wang, Jia Wei, Lai Wei, Yida Yang, Zujiang Yu, Li Zhang, Quan Zhang, Wenhong Zhang, Yuexin Zhang, Caiyan Zhao, Hong Zhao, Yingren Zhao

Members of the Guidelines Secretary Group

Yali Feng, Shan Fu, Jinfeng Liu, Naijuan Yao

Members of the Evidence Synthesis Team

Yanfang Ma, Juan Li, Xufei Luo, Changli Qian, Zhen Tian, Jianjian Wang, Yuchao Wu, Nan Yang, Jingyi Zhang, Qi Zhou

\section{Acknowledgments}

We appreciated all experts and clinicians participating in the guidelines' development, as well as the patients involved.

\section{Funding}

This work was supported by Beijing Chen Jumei Foundation, Key R\&D Program of Shaanxi (S2018-YF-ZDSF-0240), National Natural Science Foundation of China Grants (81670537, 81770594), Chinese National Research Grant of the Thirteenth Five-Year Plan for Key Projects in Infectious Diseases (13th Five Year, China; Project No. 2017ZX10202202-002006). The meeting expenses of the Guidelines Steering Committee and the Guidelines Development Panel were funded by Beijing Chen Jumei Foundation.

\section{Conflict of interest}

The authors have no conflict of interests related to this publication.

\section{Author contributions}

Study concept and design (YRZ, GQW, WHZ, HR), drafting of the manuscript (JFL, TYC), critical revision of the manuscript for important intellectual content (YLC, WHZ, GQW, HR), administrative, study supervision (YRZ, GQW, WHZ, HR).

\section{References}

[1] Global, regional, and national age-sex-specific mortality and life expectancy, 1950-2017: a systematic analysis for the Global Burden of Disease Study 2017. Lancet 2018;392:1684-1735. doi: 10.1016/S0140-6736(18)318919.

[2] World Health Organization. Global health sector strategy on viral hepatitis 2016-2021. Available from: https://apps.who.int/iris/bitstream/handle/10665/246177/WHO-HIV-2016.06-eng.pdf. Accessed at June 2016.

[3] Obayashi A, Okochi K, Mayumi M. Familial clustering of asymptomatic carriers of Australia antigen and patients with chronic liver disease or primary liver cancer. Gastroenterology 1972;62:618-625.

[4] Yang $Y$, Jin L, He YL, Wang $K$, Ma XH, Wang J, et al. Hepatitis B virus infection in clustering of infection in families with unfavorable prognoses in northwest China. J Med Virol 2013;85:1893-1899. doi: 10.1002/jmv.23649.

[5] Liu J, Yao N, Chen T, Fu S, Wu Y, Feng Y, Tian Z, et al. FA-01-Prevalence of mother-to-child transmission of hepatitis $B$ virus: A systematic review and meta-analysis. J Hepatol 2019;70: E123-E124. doi: 10.1016/S0618-8278 (19)30217-8.

[6] World Health Organization. WHO handbook for guideline development, 2nd ed. Available from: https://apps.who.int/iris/handle/10665/145714.

[7] Brouwers MC, Kho ME, Browman GP, Burgers JS, Cluzeau F, Feder G, et al. AGREE II: advancing guideline development, reporting, and evaluation in health care. Prev Med 2010;51:421-424. doi: 10.1016/j.ypmed.2010.08. 005 . 
[8] Chen Y, Yang K, Marušić A, Qaseem A, Meerpohl J], Flottorp S, et al. Ein Instrument zur Erstellung von Leitlinienberichten: das RIGHT-Statement [A reporting tool for practice guidelines in health care: the RIGHT statement. Z Evid Fortbild Qual Gesundhwes 2017;127-128:3-10. doi: 10.1016/j.zefq. 2017.10.008.

[9] Jaeschke R, Guyatt GH, Dellinger P, Schünemann H, Levy MM, Kunz R, et al. Use of GRADE grid to reach decisions on clinical practice guidelines when consensus is elusive. BMJ 2008;337:a744. doi: 10.1136/bmj.a744.

[10] Jourdain G, Ngo-Giang-Huong N, Harrison L, Decker L, Khamduang W, Tierney $\mathrm{C}$, et al. Tenofovir versus placebo to prevent perinatal transmission of hepatitis B. N Engl J Med 2018;378:911-923. doi: 10. 1056/NEJMoa1708131.

[11] Pan CQ, Duan Z, Dai E, Zhang S, Han G, Wang Y, et al. Tenofovir to prevent hepatitis $B$ transmission in mothers with high viral load. N Engl J Med 2016; 374:2324-2334. doi: 10.1056/NEJMoa1508660.

[12] Fu S, Yao N, Feng Y, Li J, Zhao Y. Dynamic changes of HBsAg and/or HBV DNA in infants born to $\mathrm{HBsAg}(+)$ mothers: A systematic review and network meta-analysis. Hepatology 2019;70:581A.

[13] Wang J, He Y, Jin D, Liu J, Zheng J, Yuan N, et al. No response to hepatitis B vaccine in infants born to $\mathrm{HBsAg}(+)$ mothers is associated to the transplacental transfer of HBsAg. Infect Dis (Lond) 2017;49:576-583. doi: 10 . $1080 / 23744235.2017 .1292541$.

[14] Chen T, Wang J, Feng Y, Yan Z, Zhang T, Liu M, et al. Dynamic changes of HBV markers and HBV DNA load in infants born to $\mathrm{HBsAg}(+)$ mothers: can positivity of HBsAg or HBV DNA at birth be an indicator for HBV infection of infants? BMC Infect Dis 2013;13:524. doi: 10.1186/1471-2334-13-524.

[15] Consensus on the management of hepatitis B. virus infection in women of childbearing age. Chinese Journal of Viral Diseases 2018;8:164-169.

[16] Management algorithm for interrupting mother-to-child transmission of hepatitis B. Journal of Clinical Hepatology 2017;33:1214-1217.

[17] Terrault NA, Lok ASF, McMahon BJ, Chang KM, Hwang JP, Jonas MM, et al. Update on prevention, diagnosis, and treatment of chronic hepatitis $B$ : AASLD 2018 hepatitis B guidance. Hepatology 2018;67:1560-1599. doi: 10.1002/hep.29800.

[18] Mast EE, Margolis HS, Fiore AE, Brink EW, Goldstein ST, Wang SA, et al. A comprehensive immunization strategy to eliminate transmission of hepatitis $B$ virus infection in the United States: recommendations of the Advisory Committee on Immunization Practices (ACIP) part 1: immunization of infants, children, and adolescents. MMWR Recomm Rep 2005;54:1-31.

[19] Immunization on schedules and instructions for vaccines of the Nationa Immunization Program (2016 version). Chinese Journal of Viral Diseases 2017;7:81-86.

[20] Fu S, Yao NJ, Feng YL, Li J, Wu YC, Tian Z, et al. The efficacy of two different dosages hepatitis $B$ immunoglobulin in interrupting mother-to-infant transmission of hepatitis $B$ virus: a systematic review and meta-analysis. Journal of Hepatology 2019;70: E124.

[21] Zhang, JY, Qi Y, Zhang TL. Vaccine response rates after immunization of infants born to HBV infection and normal pregnant women: systematic review. Papers collection of 2019 forum on evidence-based science and knowledge transfer and first symposium on clinical epidemiology and evidence-based medicine in northwest China, Lanzhou, 2019:47-48.

[22] Wen WH, Chang MH, Zhao LL, Ni YH, Hsu HY, Wu JF, et al. Mother-to-infant transmission of hepatitis $B$ virus infection: significance of maternal viral load and strategies for intervention. J Hepatol 2013;59:24-30. doi: 10.1016/j. jhep.2013.02.015.

[23] Sarin SK, Kumar M, Lau GK, Abbas Z, Chan HL, Chen CJ, et al. Asian-Pacific clinical practice guidelines on the management of hepatitis B: a 2015 update. Hepatol Int 2016;10:1-98. doi: 10.1007/s12072-015-9675-4.

[24] EASL 2017 Clinical Practice Guidelines on the management of hepatitis B. virus infection. J Hepatol 2017;67:370-398. doi: 10.1016/j.jhep.2017.03. 021.

[25] National Institute for Health and Care Excellence: Clinical guidelines. In: Hepatitis B. (Chronic): Diagnosis and management of chronic hepatitis B in children, young people and adults. National Institute for Health and Care Excellence, London, UK, 2013.

[26] Brown RS Jr, McMahon BJ, Lok AS, Wong JB, Ahmed AT, Mouchli MA, et al. Antiviral therapy in chronic hepatitis $B$ viral infection during pregnancy: $A$ systematic review and meta-analysis. Hepatology 2016;63:319-333. doi: 10.1002/hep.28302.

[27] Wiseman E, Fraser MA, Holden S, Glass A, Kidson BL, Heron LG, et al. Perinatal transmission of hepatitis $B$ virus: an Australian experience. Med J Aust 2009; 190:489-492.

[28] Zou H, Chen Y, Duan Z, Zhang H, Pan C. Virologic factors associated with failure to passive-active immunoprophylaxis in infants born to HBsAg-positive mothers. J Viral Hepat 2012;19:e18-e25. doi: 10.1111/j.1365-2893. 2011.01492.x.

[29] Sebivo (telbivudine). Product information. Novartis, Basel, Swiss, 2007.

[30] Viread (tenofovir). Product information. Gilead Sciences, Foster City, CA, USA, 2013.
[31] Han GR, Cao MK, Zhao W, Jiang HX, Wang CM, Bai SF, et al. A prospective and open-label study for the efficacy and safety of telbivudine in pregnancy for the prevention of perinatal transmission of hepatitis B virus infection. J Hepatol 2011;55:1215-1221. doi: 10.1016/j.jhep.2011.02.032.

[32] Han GR, Jiang HX, Wang CM, Ding Y, Wang G], Yue X, et al. Long-term safety and efficacy of telbivudine in infants born to mothers treated during the second or third trimesters of pregnancy. J Viral Hepat 2017;24:514-521. doi: $10.1111 /$ jvh.12670.

[33] Wang HL, Lu X, Yang X, Xu N. Antiviral therapy in lamivudine-resistant chronic hepatitis B patients: A systematic review and network meta-analysis. Gastroenterol Res Pract 2016;2016:3435965. doi: 10. $1155 / 2016 / 3435965$

[34] Wang J, Liu J, Qi C, Yan T, Cao F, Jin L, et al. Efficacy of tenofovir disoproxil fumarate to prevent vertical transmission in mothers with lamivudine-resistant HBV. Antivir Ther 2015;20:681-687. doi: 10.3851/IMP2981.

[35] Momper JD, Best B, Wang J, Stek A, Cressey T, Burchett S, et al. Tenofovir alafenamide pharmacokinetics with and without cobicistat in pregnancy. 22nd International AIDS Conference; 23-37 July 2018; Amsterdam.

[36] Wu $Y$, Liu J, Feng $Y$, Fu S, Ji F, Ge L, et al. Efficacy and safety of antiviral therapy for HBV in different trimesters of pregnancy: systematic review and network meta-analysis. Hepatol Int 2020;14:180-189. doi: 10 . 1007/s12072-020-10026-0.

[37] Wu $Q$, Huang $H$, Sun $X$, Pan $M, H e Y$, Tan $S$, et al. Telbivudine prevents vertical transmission of hepatitis $B$ virus from women with high viral loads: a prospective long-term study. Clin Gastroenterol Hepatol 2015;13:1170-1176. doi: $10.1016 / j . c g h .2014 .08 .043$.

[38] Yu $\mathrm{M}$, Jiang $\mathrm{Q}$, Ji $\mathrm{Y}$, Jiang $\mathrm{H}, \mathrm{Wu} \mathrm{K}$, Ju $\mathrm{L}$, et al. The efficacy and safety of antiviral therapy with lamivudine to stop the vertical transmission of hepatitis B virus. Eur J Clin Microbiol Infect Dis 2012;31:2211-2218. doi: 10. 1007/s10096-012-1557-2.

[39] Yang N, Kang S, Yao N. Safety of nucleoside analogues in pregant women with chronic HBV infection: a systematic review. Papers collection of 2019 forum on evidence-based science and knowledge transfer and first symposium on clinical epidemiology and evidence-based medicine in northwest China, Lanzhou, 2019:44-45.

[40] Ling $\mathrm{H}$. Ministry of Health issued the report on prevention and treatment of birth defects in China. China Modern Medicine 2012;19:1

[41] Correa A, Cragan J, Kucik J. Metropolitan Atlanta Congenital Defects Program 40th anniversary edition surveillance report: Reporting birth defects surveillance data 1968-2003 (vol 79, pg 65, 2007). Birth Defects Research Part aClinical and Molecular Teratology 2008;82:41-62. doi: 10.1002/bdra. 20434.

[42] Spearman CW, Sonderup MW, Botha JF, van der Merwe SW, Song E, Kassianides $C$, et al. South African guideline for the management of chronic hepatitis B: 2013. S Afr Med J 2013;103:337-349.

[43] World Health Organization. Guidelines for the prevention, care and treatment of persons with chronic hepatitis B. infection.

[44] Beauverd Y, Radia D, Cargo C, Knapper S, Drummond M, Pillai A, et al. Pegylated interferon alpha-2a for essential thrombocythemia during pregnancy: outcome and safety. A case series. Haematologica 2016;101:e182-e184. doi: 10.3324/haematol.2015.139691.

[45] Sakai K, Ueda A, Hasegawa M, Ueda Y. Efficacy and safety of interferon alpha for essential thrombocythemia during pregnancy: two cases and a literature review. Int J Hematol 2018;108:203-207. doi: 10. 1007/s12185-017-2397-8.

[46] Sandberg-Wollheim M, Alteri E, Moraga MS, Kornmann G. Pregnancy outcomes in multiple sclerosis following subcutaneous interferon beta-1a therapy. Mult Scler 2011;17:423-430. doi: 10.1177/1352458510394610.

[47] Coyle PK, Sinclair SM, Scheuerle AE, Thorp JM Jr, Albano JD, Rametta MJ. Final results from the Betaseron (interferon $\beta-1 \mathrm{~b}$ ) Pregnancy Registry: a prospective observational study of birth defects and pregnancy-related adverse events. BMJ Open 2014;4:e004536. doi: 10.1136/bmjopen-2013004536.

[48] Atasoy HI, Sirmatel P, Sirmatel F. Pegylated interferon therapy during early pregnancy for hepatitis B infection: does it prevent vertical transmission? J Matern Fetal Neonatal Med 2017;30:745-747. doi: 10.1080/14767058. 2016.1183639.

[49] Hiratsuka M, Minakami H, Koshizuka S, Sato I. Administration of interferonalpha during pregnancy: effects on fetus. J Perinat Med 2000;28:372-376. doi: $10.1515 /$ JPM.2000.047.

[50] Labarga P, Pinilla J, Cachorro I, Ruiz Y. Infant of 22 months of age with no anomalies born from a HCV- and HIV-infected mother under treatment with pegylated interferon, ribavirin and antiretroviral therapy during the first 16 weeks of pregnancy. Reprod Toxicol 2007;24:414-416. doi: 10.1016/j. reprotox.2007.07.002.

[51] Romero RS, Lünzmann C, Bugge JP. Pregnancy outcomes in patients exposed to interferon beta-1b. J Neurol Neurosurg Psychiatry 2015;86:587-589. doi: 10.1136/jnnp-2014-308113.

[52] Chang CY, Aziz N, Poongkunran M, Javaid A, Trinh HN, Lau D, et al. Serum alanine aminotransferase and hepatitis B DNA flares in pregnant and 
postpartum women with chronic hepatitis B. Am J Gastroenterol 2016;111: 1410-1415. doi: 10.1038/ajg.2016.296.

[53] Yi W, Pan CQ, Li MH, Wan G, Lv YW, Liu M, et al. The characteristics and predictors of postpartum hepatitis flares in women with chronic hepatitis $B$. Am J Gastroenterol 2018;113:686-693. doi: 10.1038/s41395-018-0010-2.

[54] Liu J, Wang J, Qi C, Cao F, Tian Z, Guo D, et al. Baseline hepatitis B virus titer predicts initial postpartum hepatic flare: A multicenter prospective study. J Clin Gastroenterol 2018;52:902-907. doi: 10.1097/MCG. 0000000000000877.

[55] The guideline of prevention and treatment fro chronic hepatitis B: a 2015 update. Chinese Journal of Infectious Diseases 2015;33:641-662.

[56] Fu S, Yao N, Liu J. Meta-analysis of changes in HBV DNA levels in pregnant women with chronic HBV infection after antiviral intervention. Papers collection of 2019 forum on evidence-based science and knowledge transfer and first symposium on clinical epidemiology and evidence-based medicine in northwest China, Lanzhou, 2019:54-55.

[57] Marcellin P, Gane EJ, Flisiak R, Trinh HN, Petersen J, Gurel S, et al. Long term treatment with tenofovir disoproxil fumarate for chronic hepatitis $B$ infection is safe and well tolerated and associated with durable virologic response with no detectable resistance: 8 year results from two phase 3 trials. Hepatology 2014;60:313A-314A.

[58] Liaw YF, Gane E, Leung N, Zeuzem S, Wang Y, Lai CL, et al. 2-Year GLOBE trial results: telbivudine Is superior to lamivudine in patients with chronic hepatitis B. Gastroenterology 2009;136:486-495. doi: 10.1053/j.gastro.2008. 10.026 .

[59] Lin J, Sha Y, Qiu P. Effects of hepatitis B virus infection in women with different ovarian reserve on outcomes of in vitro fertilization and embryo transfer. Reproduction and Contraception 2017;37:106-110.

[60] Yu J. The effect of female HBV infection on the outcome of in vitro fertilization-embryo transfer. Kunming Medical University 2015.

[61] Liu L, Liu Q, Wen Y. Ovarian reserve function in infertile women with HBV infection. Journal of Reproductive Medicine 2016;25:27-31.

[62] Yao N, Ma Y, Wang J, Zhang X, Zhao Y, Chen Y, et al. Role of infertile female chronic hepatitis $B$ virus infection in assisted reproduction and infantile outcomes: A systematic review and meta-analysis. Hepatology 2019;70:599A

[63] Tian Z, Li J, Liu J, Chen T, Zhao Y. Caesarean section versus vaginal delivery to prevent mother-to-child transmission of hepatitis $B$ virus: A meta-analysis. J Hepatol 2019;70:E124. doi: 10.1016/S0618-8278(19)30219-1.

[64] Tran TT, Ahn J, Reau NS. ACG clinical guideline: Liver disease and pregnancy. Am J Gastroenterol 2016;111:176-194; quiz 196. doi: 10.1038/ajg.2015. 430.

[65] Giles M, Visvanathan K, Lewin S, Bowden S, Locarnini S, Spelman T, et al. Clinical and virological predictors of hepatic flares in pregnant women with chronic hepatitis B. Gut 2015;64:1810-1815. doi: 10.1136/gutjnl-2014308211.

[66] Liu J, Wang J, Jin D, Qi C, Yan T, Cao F, et al. Hepatic flare after telbivudine withdrawal and efficacy of postpartum antiviral therapy for pregnancies with chronic hepatitis B virus. J Gastroenterol Hepatol 2017;32:177-183. doi: 10.1111/jgh.13436.

[67] Zhao $Y$, Feng $Y$, Wu $Y$, Ji $F$, Chen $T$. Hepatic flare after antiviral treatment withdraw in post-partum for pregnancy of chronic hepatitis $B$ viral infection A pairwise and Bayesian network meta-analysis. J Hepatol 2019;70:E127. doi: 10.1016/S0618-8278(19)30224-5.

[68] Wang X, Lu J, Wu Y. Clinical study on liver function, virology, serological changes and the safety of drug withdrawal in pregnant women who are chronic HBV carriers during pregnancy and postpartum. Chinese Journal of Hepatology 2019;27:261-266.

[69] Zhang S, Li B, Wang X. Significance and correlation analysis of postpartum serum, saliva and breast HBV-DNA loads in parturients with different HBV infection patterns. Maternal and Child Health Care of China 2019;34:19741977.

[70] Montoya-Ferrer A, Zorrilla AM, Viljoen J, Molès JP, Newell ML, Van de Perre $\mathrm{P}$ et al. High level of HBV DNA virus in the breast milk seems not to contraindicate breastfeeding. Mediterr J Hematol Infect Dis 2015; 7:e2015042. doi: 10.4084/MJHID.2015.042.

[71] Huang $\mathrm{H}$. Effect of different feeding methods on mother-to-child transmission of hepatitis B virus infection. Chinese Hepatology 2019;24:477-478.

[72] Feng Y, Qian C, Yao N, Wu Y, Ma Y, Ma M, et al. The safety of breast-feeding on infant transmission of hepatitis $B$ virus after combined immunoprophylaxis: A systematic review and meta-analysis. Hepatology 2019;70:598A-599A.

[73] Mugwanya KK, Hendrix CW, Mugo NR, Marzinke M, Katabira ET, Ngure K, et al. Pre-exposure prophylaxis use by breastfeeding HIV-uninfected women: A prospective short-term study of antiretroviral excretion in breast milk and infant absorption. PLoS Med 2016;13:e1002132. doi: 10.1371/journal. pmed.1002132.

[74] Mirochnick M, Taha T, Kreitchmann R, Nielsen-Saines K, Kumwenda N, Joao $E$, et al. Pharmacokinetics and safety of tenofovir in HIV-infected women during labor and their infants during the first week of life. J Acquir Immune Defic Syndr 2014;65:33-41. doi: 10.1097/QAI.0b013e3182a921eb.

[75] Waitt C, Olagunju A, Nakalema S, Kyohaire I, Owen A, Lamorde M, et al. Plasma and breast milk pharmacokinetics of emtricitabine, tenofovir and lamivudine using dried blood and breast milk spots in nursing African mother-infant pairs. J Antimicrob Chemother 2018;73:1013-1019. doi: $10.1093 / \mathrm{jac} / \mathrm{dk} \times 507$. 